\title{
Ulteriori considerazioni sulla correzione topografica nei rilievi gravimetrici geominerari
}

\author{
MI. BERNAHINI (*)
}

Ricevuto il 30 Settembre 1963

Riassunto. -- Si prende in esame l'errore residuo dovuto al considerare limitati da nn piano orizzontale i compartimenti cilindrici di aleme configurazioni per le correzioni topografiche di rilievi gravimetriei.

Dopo una prima analisi delle variazioni degli errori residui in $n$ eonlpartimento, in funzione della quota media. pendenza media ed orientazione della pendenza della superficie topografica compresa nel compartimento, si considerano gli errori residui complessivi di ma zona o complesso di zone e si verifiea, mediante ma analisi operata su alcune stazioni di un rilievo, che essi sono legati alla distribuzione statistica delle pendenze.

Si suggeriseono alemi metodi speditivi per valutare in primissima approssimazione l'entita dell'errore residno e per eliminarlo di aleuni casi.

SUMMARY. - In the present paper we are dealing with residual error ocenring when considering surfaces of eylindrieal compartment in gravimetrie topographic correction, horizontal.

It is shown how residual error variations in a compartment depend on the average level, arerage slope and slope orientation of topographice surface in the eompartment.

The residual error of a zone or an ensemble of zones may be positive or negative according to statistical distribution of slope orientations. As an example, the results of a gravimetric survey are related.

Rapid methods to value the residual error and to eliminate it in sime mases are suggested.

\section{PREMESSA.}

In una precerlente nota $\left.{ }^{1}\right)$ si era posta in risalto l'accresciuta inportanza di un affinamento dei metodi in uso pere efetetuare la correzione topografica soprattutto nel caso di rilievi greominemari.

(*) Istituto di Geofisiea Mineraria - Facolta d'Ingegneria - Universita degli Studi - Roma. 
Eno dei problemi, nella adozione del metodo di correzione pii rispondente per ogni riliero, è quello della scelta della schematizzazione geometriea da impiegare. I ceriteri di seelta sono orvianente la semplieita - la rapidita del metodo e l'applossimazione che esso permette. Cio porta a dover valutare, volta per volta, l'entita degli errori che le schematizzazioni introducono.

I metodi di correzione oggi pii largamente impiegati, schematizzano il terreno assimilandolo a compartimenti cilindrici limitati superiomente da porzioni di piano aventi quota uguale a quella media della superficie topografical, che ciasceuno di essi lanechinde. Tale "spianamento n della superficie topografica e sicummente rispondente nel caso di rilievi in aree pianegrgianti quali quelle rehe si riscontrano nei grandi bacini di seclimentazione o in zone penepianate (sceudo camadese, seudo baltieo, ece.). Esso puo fornire ancora risultati sufficientemente approssimalti su altopiani spezzati in grandi blocchi da faglie (tipo altopiani africani, americani, ece.); in questi casi dovmano essere corretti, ricorrendo ad espressioni particolari, solo i eompartimenti che ricadono a cavallo di linee di dislocazione.

Esistono pero molte altre aree nelle quali tale schematizzazione non e sicuramente la piu sodelisfacente in quanto la superficie topografiea dei compartimenti non e approssimabile a porzioni di piano orizzontale.

In tali easi una valutazione degli errori che tali schematizzazioni introducono non e facile ne immediata, data soprattutto la grande variabilitì di forme ed andamenti che la superficie terrestre presenta. D'altra parte la valutazione dell'errore e, come si e detto, neeessaria per una sceelta della schenatizzazione piu idonea a ciascum easo.

Il problema in questione e stato gia affrontato da vari autori. Tra questi si ricorda J. Solaini $\left({ }^{9}\right)$, ehe pone a base di wna sua proposta sehematizzazione ma valutazione dell'ordine di grandezza di errori derivanti da particolari ipotesi di andamento della superficie topografica.

P. Tejay (7) e suceessivamente M. Cunietti $\left(^{(3)}\right.$ hanno impostato nelle linee generali il problema, cui Cunietti stesso ha dato ma risoluzione con particolare riferimento alle correzioni per le zone piu lontane della suddivisione di Fayford.

Nella presente nota si vuole portare un contributo allo studio del problema della valutazione degli errori che si commettono quando si sostituisee, agli effetti del]a correzione, una superficie orizzontale ad una superficio inclinata di ugual quota media, nel caso di eompartimenti rilindrici prossimi alla stazione (distanzo minori di $5 \mathrm{~km}$ ). 
Si considerelanno in particolare i casi in rui una schematizzazione pii approssimata alla realta sia pintrosto quella di superfiei inclinate monoclinali (*)

Una tale situazione si verifica anche in molte zone d'Italia, ad es. in quelle in eui per effetti tettonici la superfecie topografica risulta costituita da porzioni di superfici inclinate pio o meno piane fad es. nell'Appennino umbro-marchigiano). La schematizzazione indicala puo esssere rispondente anche in zono in eui l'erosione, agendo su terreni di scarsa consistenzal, ha generato vallate piu o meno ampie, molto spesso con forme a $V$ a versunti all'incirca piani.

Come si vedra in seguito, anche molti altri casi cli superfici non monoclinali sono riducibili, agli effetti delle correzioni, a superfiei monoclinali.

Per valutare l'erore che si commette nei casi citati con le correzioni usuali (errore che verri indicato come erore residuo), è opportuno promettere aleune considerazioni sulle variazioni degali errori ressidui stessi con i valori della pendenza o con le orientazioni (***) delle pendenze stesse, relativi alla superficie topografica racehiusa in un compartimento, ed al variare delle quote medie.

Successivamente si riporteramo i risultati di una indagine statistica sulle distribuzioni delle pendenze e sughi errori residui in man zona della Toscana e si trarranno, a conclusione, aleme considerazioni sul morlo di valutare a ridurre, se necessatio, gli errori resichui.

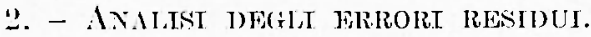

Si e promesso che si sarebbero presi in considerazione compartimenti con superficie topografica monoclinale.

Per avere una prima idea sulle variazioni degli emori residui, è sufficionte, seguendo lindirizzo gia adottato da P. Lejay (j) e da M. Cunietti $\left({ }^{3}\right)$, considerare compartimenti limitati da superfici le cui pendenze siano orientate in ogni punto o pauallelamente o normalmente alla rongiungente il centro dol compartinento con il punto di stazione. Le

$\left(^{*}\right)$ Si considera sempre la porzione di superficie topografica ricadente in un compartimento.

(**) Per "orientazione della pendenza " si intende qui la orientazione, rispetto alla stazione di misura, della direzione della massima pendenza prevalente della superficie topografica. 
orientazioni intermedie damamo effetti compresi tra quelli dovuti alle due schematizzazioni proposte.

Si suppone cioe che la superficie topografica possa essere rappresentabile: 1) eon una porzione di cono con asse verticale passante per il punto di stazione; 2) con una porzione di elicoide retto a passo costante anche esso con asse verticale passante per il punto di stazione.

Consideriamo nn sistema di coordinate cilindriche $R, a, z$, con il centro nel punto di stazione a l'asse $z$ verticale; nella prima schematizzazione le quote variano linearmente con il raggio $R$, nella seconda con l'anomalia $a$.

Indichiamo con $R_{1}, R_{2}$ ed $R_{m}$ il laggio minimo, massimo e medio ("*) del comportamento in esame linitato tra le anomalie $a_{1}$ ed $\alpha_{2}$ (Fig. 1), con $H$ la quota generiea relativa a quella del punto di stazione, con $K$ la costante di gravitazione universale e con $\sigma$ la densita.

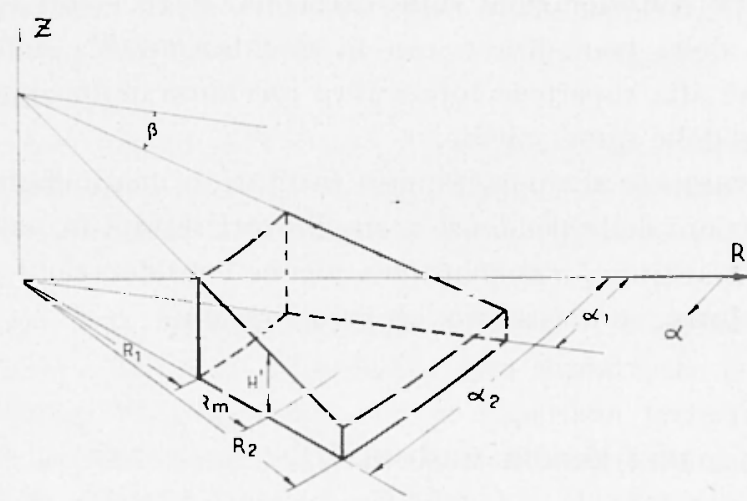

Fig. 1 - Schena di compartimento con variazione lineare delle quote con il ragrio.

Sel primo caso (quote valianti lineamente con il laggoio) indicando ron $I^{\prime}$ la quota relativa corrispondete al magio medio $R_{m}$, si può porre

$$
H=H^{\prime}+\operatorname{tg} \beta\left(K-R_{m}\right)
$$

in cui tg p e la pendenza della superficie lungo il laggoio.

$\left(^{*}\right)$ Inteso come media aritmetica dei raggi estremi: $\left(R_{1}+R_{2}\right) / 2$. 
ULTERIORI CONSIDERAZIONI SULLA CORREZIONE TOPOGRAFICA, ECC. 289

L'attrazione di tale compartimento sara: (Vedi anche L. Solaini $\left({ }^{9}\right)$ )

$$
\begin{aligned}
g & =K \sigma \int_{a_{1}}^{a_{2}} \int_{\dot{R}_{1}}^{R_{2}} \int_{0}^{H^{\prime}+\operatorname{tg} \beta\left(R-R_{m}\right)} \frac{z R}{\left(z^{-}+R^{-}\right)^{3 / 2}} d a d z d R= \\
& =-K \sigma\left(\alpha_{2}-\alpha_{1}\right) \frac{\sqrt{p^{2} R_{0}^{2}+\operatorname{tg} \beta q R_{2}+q^{2}}}{n_{n^{2}}}-
\end{aligned}
$$

$-\frac{\operatorname{tg} \beta q}{p^{3}} \ln \left[2 p \sqrt{p^{2} R_{2}^{2}+2 \operatorname{tg} \beta q R_{2}+q^{2}}+2 R_{2} p^{2}+2 \operatorname{tg} \beta q\right]-$

$-\frac{\sqrt{p^{2} R_{1}^{2}+2 \operatorname{tg} \beta q R_{1}+q^{2}}}{p^{2}}+\frac{\operatorname{tg} \beta q}{p^{3}} \ln \left[2 p \sqrt{p^{2} R_{1}^{2}+2 \operatorname{tg} \beta q R_{1}+q^{2}}+\right.$

$$
\left.\left.+2 R_{1} p^{2}+2 \operatorname{tg} \beta q\right]-\left(R_{2}-R_{1}\right)\right\}
$$

in cui

$$
p=\sqrt{1+\operatorname{tg}^{2} \beta} \quad \text { e } q=H^{\prime}-\operatorname{tg} \beta R_{m} .
$$

Nel secondo caso (quote varianti linearmente con la anomalia), indicando con $\alpha_{m}$ e con $H^{\prime \prime}$ rispettivamente l'azimut medio e la quota re-

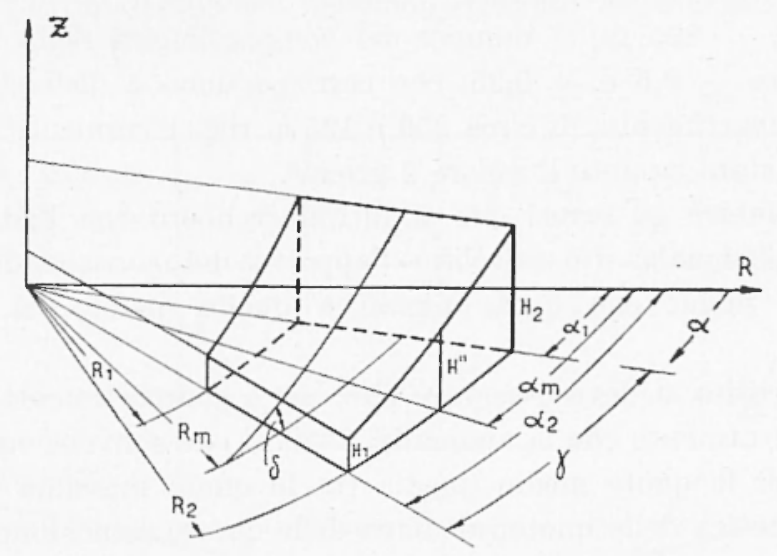

Fig. 2 - Schema di compartimento con variazione lineare delle quote con l'anomalia.

lativa ad esso, con $\operatorname{tg} \delta$ la pendenza della superficie topografica sulla circonferenza media di raggio $R_{m}$ (Fig. 2), si può porre:

da cui si ricava che

$$
H=\operatorname{tg} \delta R_{m} a
$$

$$
a_{m}=H^{\prime \prime} / \operatorname{tg} \delta R_{m}
$$


L'attrazione di tale comportamento sara:

$$
\begin{aligned}
g= & \int_{a_{1}}^{\alpha_{2}} \int_{0^{\prime}}^{\operatorname{tg} \delta R_{m} \alpha} \int_{R_{1}}^{R_{2}} \frac{z R}{\left(z^{2}+R^{-3}\right)^{3} / 2} d \alpha d z d R=\frac{1}{2} K \sigma \gamma\left[2\left(R_{2}-K_{1}\right)+\right. \\
+ & \frac{1}{2}\left(\sqrt{H_{2}^{2}+R_{1}^{2}}+\sqrt{H_{1}^{2}+R_{1}^{2}}\right)+\frac{H^{\prime \prime}}{D}\left(\sqrt{H_{2}^{2}+R_{1}^{2}}-\sqrt{H_{1}^{2}+R_{1}^{2}}\right)+ \\
& +\frac{R_{1}^{2}}{D} \ln \frac{H_{2}+\sqrt{H_{2}^{2}+R_{1}^{2}}}{H_{1}+\sqrt{H_{1}^{2}+R_{1}^{2}}}-\frac{1}{2}\left(\sqrt{H_{2}^{2}+R_{2}^{2}}+\sqrt{H_{1}^{2}+R_{2}^{2}}-\right. \\
& \left.-\frac{H^{\prime \prime}}{D}\left(\sqrt{H_{2}^{2}+R_{2}^{2}}-\sqrt{H_{1}^{2}+R_{2}^{2}}\right)-\frac{R_{2}^{2}}{D} ? \frac{H_{0}+\sqrt{H_{2}^{2}+R_{n}^{2}}}{H_{1}+\sqrt{H_{1}^{2}+R_{2}^{2}}}\right]
\end{aligned}
$$

in cui $\gamma=u_{2}-\omega_{1}$ e l'ampiezza angolare del compartimento, $H_{0}$ ed $H_{1}$ le quote relative massima e minima (date da $H^{\prime \prime} \pm R_{n} \operatorname{tg} \delta \cdot \gamma / 2$ ) e $D$ il dislivello $H_{2}-H_{1}$.

I valori forniti dalle espressioni [1] e [?] sono stati calcolati, al variare delle quote $H^{\prime}$ ed $Z^{\prime \prime}$ rispettivamente, per un compartimento della zona $F^{\prime}$ della configurazione di Hammer ( ${ }^{5}$ ) (raggio minimo $R_{1}=390 \mathrm{~m}$; raggrio massimo $k_{2}=895 \mathrm{~m}$; il numero dei compartimenti della zona e 8), per pendenze $\pm 0,5$ e $\pm 0,25$, che corrispondono a dislivelli massimi entro il compartimento, di circa 250 e $125 \mathrm{~m}$ rispettivamente (*). Per la densita $\sigma$ e stato assunto il valore $2 \mathrm{gr} / \mathrm{cm}^{3}$.

Per valutare gli errori che si introdurrebbero con l'adozione del metodo tradizionale, si e calcolato l'apporto del corrispondente compartimento piano con quote uguali a quella media del compartimento.

In proposito si deve osservare che, per i compartimenti con quota variabile linearmente con la anomalia, i valori che convenzionalmente si adottano per le quote medie (media tra le quote massima e minima, media aritmetica delle quote, mediana delle quote) coincidono tutte con il valore $H^{\prime \prime}$ sopra indicato. Per i compartimenti con quote variabili linearmente con il raggio, invece, le tre medie non sono coincidenti e pertanto ciascuma di esse porta a risultati diversi. In questo caso gli errori sono stati calcolati facendo riferimento al compartimento oriz-

$\left(^{*}\right)$ i da notare che nel compartimento considerato sussiste la relazione $R_{m} \cdot \eta \simeq R_{2}-R_{1}$ e pertanto $\mathrm{i}$ dislivelli risultano circa gli stessi nei due casi. 


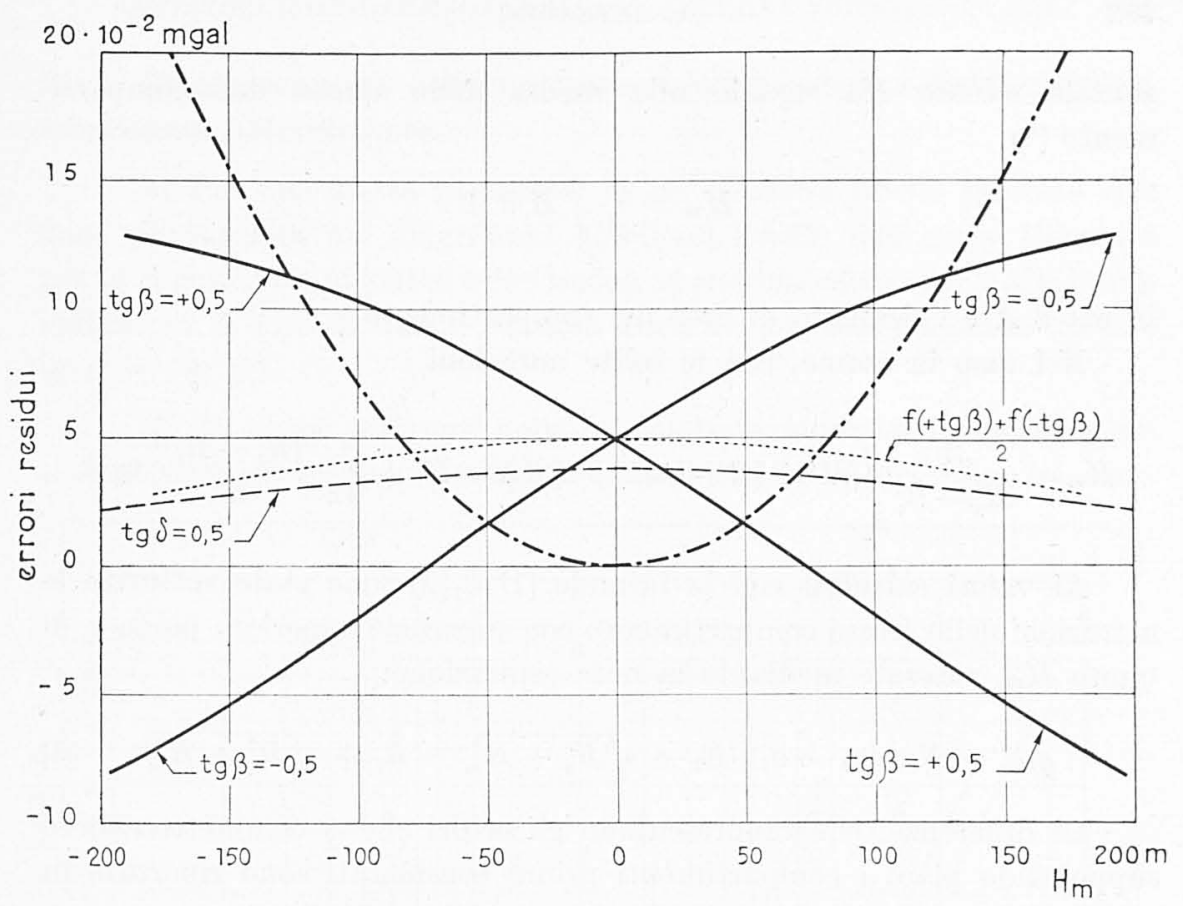

Fig. $3 a$ - Errori residui per un compartimento della zona $F$ di Hammer per pendenze 0,5 .

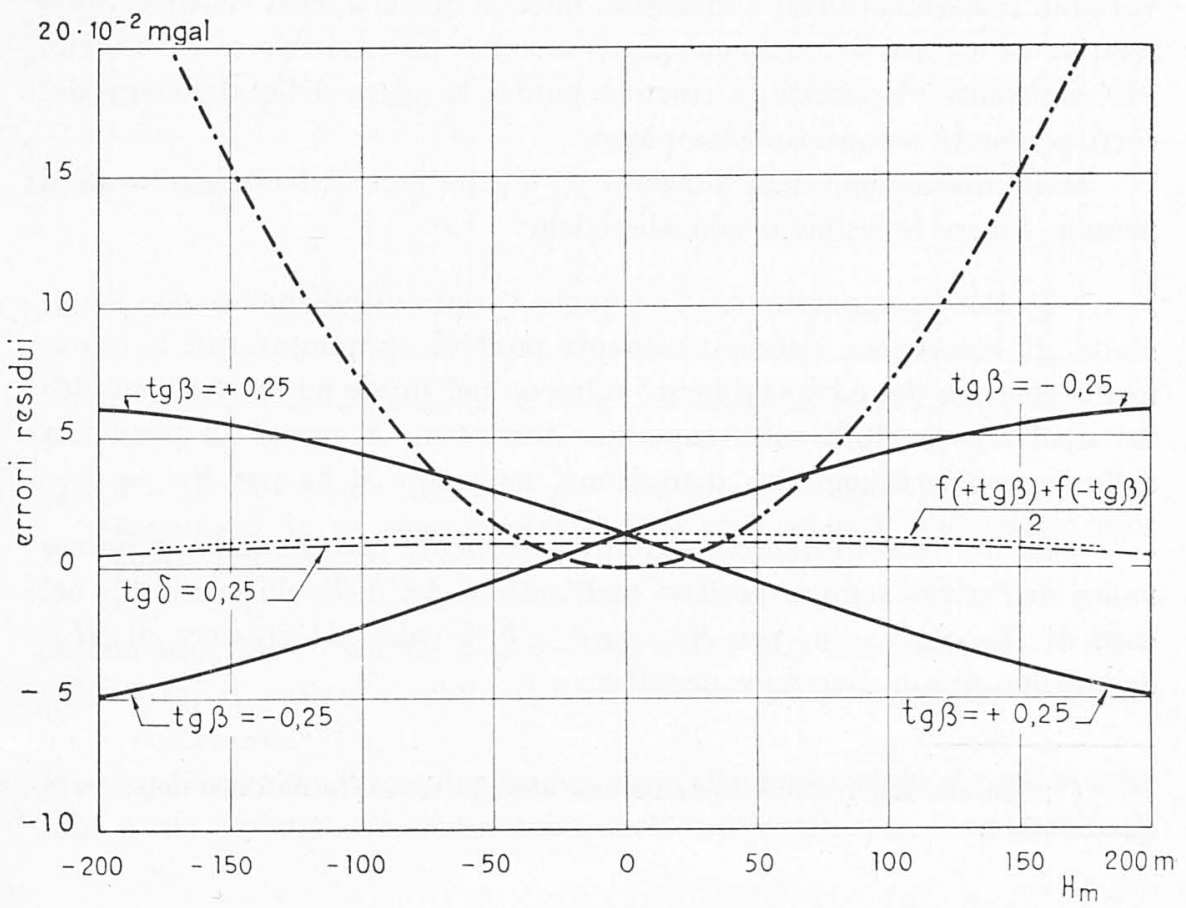

Fig. $3 b$ - Errori residui per un compartimento della zona $F$ di Hammer per pendenze 0,25 . 
zontale avente $H_{m}$ uguale alla media delle quote del compartimento $(*)$

$$
H_{m}=\frac{1}{A} \int_{d} H d A
$$

in cui $d A$ e l'elemento di area del compartimento.

Nel caso in esame, con le solite notazioni,

$$
H_{m}=\frac{2}{R_{2}^{2}-R_{1}^{2}} \int_{\dot{R}_{1}}^{R_{2}}\left[H^{\prime}+\left(R-R_{m} \operatorname{tg} \beta\right] R d R=H^{\prime}+\frac{1}{12} \frac{\left(R_{2}-R_{1}\right)^{2}}{R_{m}} \operatorname{tg} \beta\right.
$$

Ai valori calcolati con le formule [1] e [2] sono state sottratte le attrazioni dello stesso compartimento con superficie superiore piana e di quota $H_{m}$, ricavate mediante la nota espressione:

$$
g_{H_{m}}-K \sigma\left(a_{2}-a_{1}\right)\left(R_{2}-\sqrt{R_{2}^{2}+H_{m}^{2}}-R_{1}+\sqrt{R_{1}^{2}+H_{m}^{2}}\right.
$$

Le differenze, che rappresentano gli errori che si commetterebbero supponendo piani i compartimenti prima considerati sono riportate in Figura 3, separatamente per le pendenze 0,25 e 0,50 .

Le due curve a tratti sono relative ai compartimenti con quote variabili linearmente con l'anomalia, mentre quelle a linea continua sono relative ai compartimenti con quote variabili linearmente con il raggio. Per confronto e riportata, a tratto e punto, la curva delle attrazioni del corrispondente compartimento piano.

Dalla discussione delle funzioni [1] e [2] e dei relativi diagrammi si possono trarre le seguenti considerazioni:

1) Per i compartimenti con quote variabili linearmente con l'anomalia, gli errori sono sistematicamente positivi, comunque vari la quota media relativa del compartimento (almeno per quote relative nell'ambito dei dislivelli possibili sulla superficie terrestre). A parita di pendenza della superficie topografica il massimo dell'errore si ha per $H_{m}=0$.

2) Nel caso di quote variabili linearmente con il raggio, si hanno valori dell'errore sempre positivi (nell'ambito dei dislivelli possibili), nel caso di $H_{m} \cdot \operatorname{tg} \beta<0$; per $H_{m} \cdot \operatorname{tg} \beta>0$, i valori al crescere di $\left.\mid H_{m}\right\}$ decrescono fino a diventare negativi.

$\left(^{*}\right)$ Questa del resto e la tendenza attuale derivata dall'uso delle carte quadrettate. 
3) Al decrescere delle pendenze, i valori assoluti degli errori diminuiscono notevolmente.

4) Per una stessa pendenza $|\operatorname{tg} \beta|$, la curva media indicata con linea punteggiata nei diagrammi di Figura 1 delle due curve calcolate per $\operatorname{tg} \beta$ positivo e negativo e con buona approssimazione simile alla curva valida per il caso di quote variabili linearmente con lanomalia quando $\operatorname{tg} \delta=|\operatorname{tg} \beta /|$.

5) Gli errori derivanti dalla schematizzazione classica possono essere notevoli ed in alcuni casi non tollerabili. Ad es. nel caso del com-

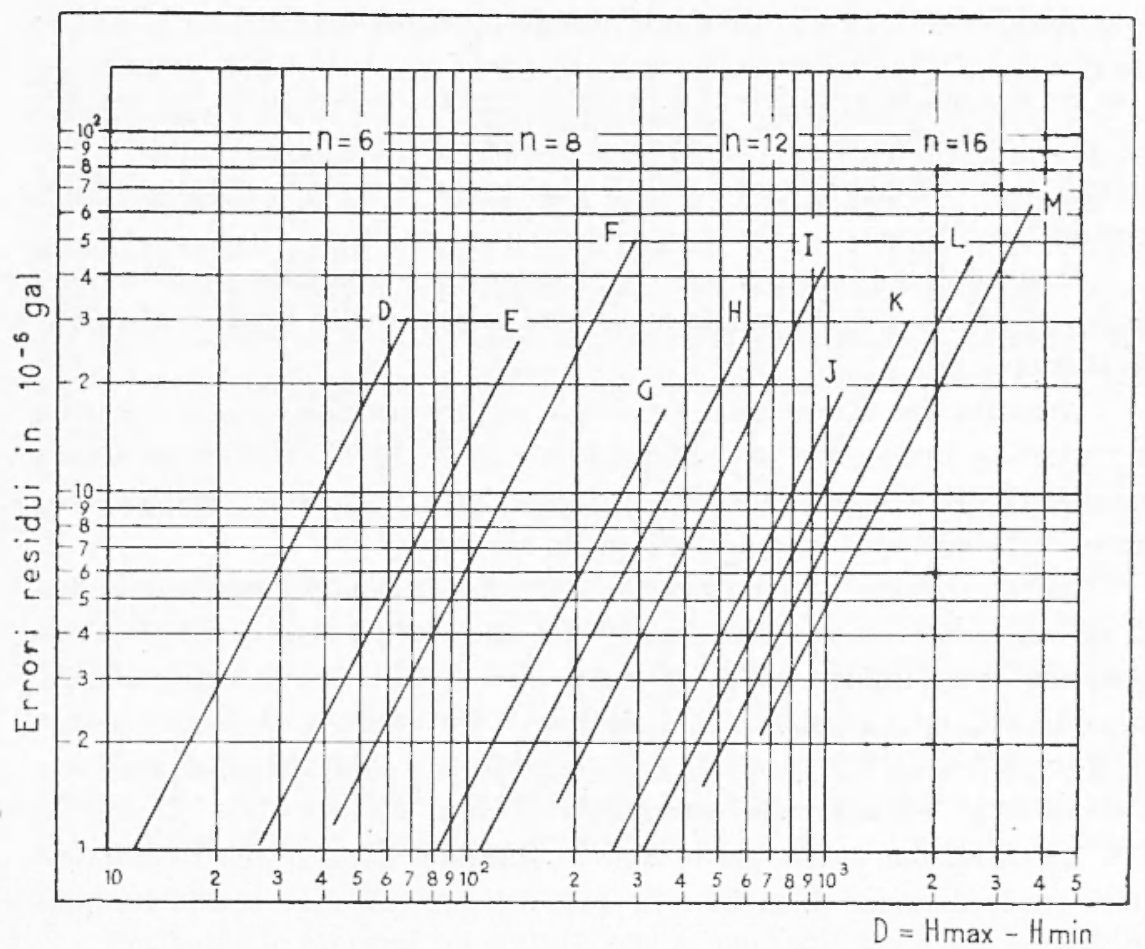

Fig. 4 - Errori residui massimi per variazione lineare delle quote con l'anomalia, in un compartimento delle varie zone di Hammer in funzione del dislivello massimo nel compartimento.

partimento citato, per $\operatorname{tg} \beta=-0,5$ e per $H_{m}$ compresi tra -50 e $+12 \tilde{5} \mathrm{~m}$, il valore assoluto dell'errore residuo è maggiore di quello dellis correzione.

Per fornire un ordine di grandezza di tali errori residui al variare delle zone e del dislivello massimo nei singoli compartimenti, sono stati cal- 
colati i valori degli errori, per un compartimento generico delle varie zone della configurazione di Hammer, mediante la espressione [2], supponendo $H_{m}=0$. I valori degli errori sono riportati nella Figura 4 .

6) Al variare della orientazione della pendenza media della superficie topografica, gli errori, soprattutto nel caso di valori assoluti $H_{m}$ grandi, variano in una gamma molto ampia.

3. - MNALISI DEgLI ERRORI RESIDUI DI UN COMPLESSO DI ZONE IN FUNZIONE DELLA DISTRIBUZIONE DELLE PENDENZE.

Se si considera un'intera zona od un complesso di zone, l'errore residuo complessivo sara ovviamente la somma degli errori residui dei singoli compartimenti; errori questi che, come si e visto, possono essere negativi o positivi.

Il segno dell'errore residuo complessivo dipendera dalla distribuzione delle orientazioni delle pendenze e delle quote medie intorno al punto di stazione.

A parita del valore della pendenza, se prevarmnno i compartimenti a variazione delle quote con il raggio con $H_{m} \cdot \operatorname{tg} \beta>0$, l'errore totale residuo risultera negativo, cioe il valore della correzione calcolato con le normali Tabelle sara maggiore di quello esatto.

Per es. consideriamo il caso limite di una stazione situata sulla vetta di un rilievo isolato rappresentabile con un cono ad esse verticale. Supponiamo che il rilievo abbia un diametro uguale a quello esterno della zona I della configurazione di Hammer $\left(^{5}\right.$ ) (n 8938) e pendenza nedia, $\operatorname{tg} \beta$, uguale al $20 \%$. La quota della somnita su cui e situata la stazione e di m 893,8 rispetto alla pianura circostante.

La correzione esatta per le zone di Hammer dalla $D$ alla $I$ comprese (cioe tra le distanze di m 53 e m 4469) si puo calcolare con la formula di Joung

$$
g=2 \pi k \sigma\left(R_{2}-R_{1}\right)(1-\cos \beta)
$$

e risulta di 7,18 mgal.

La correzione calcolata con le Tabelle di Hammer è di 8,27 mgal; l'errore residuo è dunque di - 1,09 mgal.

Un caso analogo a quello citato e quello di una stazione situata nel fondo di una grande vallata; tutti i compartimenti delle zone che risultano comprese nella vallata avranno prevalente variazione delle quote 
con il raggio (*) con $H_{m}>0$ e $\operatorname{tg} \beta>0$. Gli errori residui saranno percio anche qui in massima parte negativi.

Anche nel caso di stazioni situate su di un pendio, per tutte le zone che ricadono interamente sul pendio stesso, si avra una prevalenza di compartimenti con $\operatorname{tg} \bar{\beta} \cdot H_{n}>0$ e quindi un errore residuo complessivo negativo. Cio si verifica molto spesso per le zone piu vicine alla stazione.

Non appena si considerano zone che interessano aree al di fuori del pendio sul quale e situata la stazione, si potranno verificare compartimenti con $H_{m} \cdot \operatorname{tg} \beta<0$ e quindi con errori residui notevoli e positivi. L'errore residuo di tali zone potra essere allora positivo o negativo.

Se si considerano zone via ria piu lontane, vi sara sempre maggiore probabilità che le pendenze medie dei vari compartimenti siano orientate casualmente, cioe che nel complesso ad un compartimento con $\operatorname{tg} \beta$. - $H_{m}<0$ ne corrisponda un altro con uguali valori assoluti delle quote medie relative e delle pendenze, ma con $H_{m} \cdot \operatorname{tg} \beta>0$. In tal caso la somma dei due errori è un numero positivo (**). Se si considerano poi anche i compartimenti con variazione prevalentemente angolare delle quote, il cui crrore residuo è sistematicamente positivo, si possono ottenere errori complessivi positivi di non trascurabile entita.

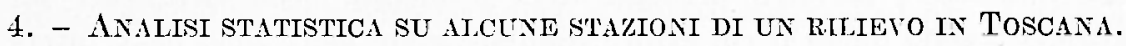

Un esempio nel quale si e potuto rilevare la validita delle considerazioni esposte nel paragrafo precedente è fornito dai risultati di un rilievo gravimetrico regionale della zona del Monte Amiata, gia ricordato nella nota citata $\left({ }^{* * * *}\right)\left({ }^{1},{ }^{4}\right)$.

Si riportano dapprima i risultati di una analisi statistica della distribuzione delle orientazioni delle pendenze prevalenti relative ai compartimenti delle zone dalla $D$ alla $J$ della configurazione di Hammer per alcune stazioni del rilievo stesso.

Fra tutte le stazioni sono state scelte, per ogni taroletta al 25.000 dell'I.G.M., tre stazioni situate verso il centro delle tarolette stesse.

$\left(^{*}\right)$ Si eceettuano i compartimenti ricadenti nel fondo della vallata nei quali la variazione delle quote risulta prevalentemente angolare.

(**) La curva media delle due correzioni assume sempre valori positivi come indicato dalle linee punteggiate della Fig. 3.

$\left({ }^{* * *}\right)$ In tale rilievo si era cereato di situare le stazioni in luoghi quanto possibile pianeggianti o piani. Pertanto le prime zone (almeno fino a $200 \mathrm{~m}$ dalle stazioui) ricadono in casi di prevalente variazione radiale delle quote con $\operatorname{tg} \beta \cdot H_{n}>0$. 
Le stazioni prese in considerazione sono state complessivamente $52\left(^{*}\right)$. Per ogni compartimento delle varie zone e stata valutata la orientazione prevalente delle pendenze della superficie topografiea; in base a cio i compartimenti sono stati classificati in 6 gruppi:

gruppo 1: compartimenti a superficie pianeggiante o quasi pianeggiante; gruppo 2: compartimenti con variazioni delle quote prevelentemente radiali con $H_{m} \simeq \mathbf{0}$;

gruppo 3: compartimenti con variazioni delle quote prevalentemente radiali ma con pendenze ugualmente distribuite nei due sensi;

gruppo 4: compartimenti con variazioni delle quote prevalentemente radiali con $H_{m} \cdot \operatorname{tg} \beta>0$;

gruppo 5: compartimenti con variazioni delle quote prevalentemente radiali con $H_{m} \cdot \operatorname{tg} \beta<0$;

gruppo 6: compartimenti con variazioni delle quote prevalentemente angolari.

Per avere una idea immediata sugli errori residui da attribuirsi ai singoli gruppi, si possono riprendere in esame i diagrammi di Fig. 3. Non si considerano ovviamente i compartimenti del gruppo 1 che non debbono essere corretti; per ogni data pendenza i compartimenti del gruppo 2 sono quelli il cui errore residuo ricade nella zona delle intersezioni delle curve degli errori residui; quelli del gruppo 3 sono quelli il cui errore residuo ricade sulla curva mediana punteggiata; quelli del gruppo 4 sui due rami piu bassi dei diagrammi; quelli del gruppo 5 sui due rami piu alti; quelli del gruppo 6 sulla curva $\operatorname{tg} \delta=$ cost.

Per una analisi globale, i compartimenti a prevalente variazione radiale delle quote sono suddivisi in due gruppi indicati con le lettere $A$ e $B$. Nel gruppo $A$ si sono posti tutti i compartimenti del gruppo 4 e la meta di quelli dei gruppi 2 e 3 ; nel gruppo $B$ quelli del gruppo 5 e l'altra meta di quelli dei gruppi 2 e 3 (vedi Tabella I a pag. seguente).

Osservando tale Tabella si nota che, passando a zone via via più lontane, il rapporto $A / B$ diminuisce pur mantenendosi sempre superiore all'unita. Mentre per la zona $E$ esso e 4,14 , gia nella zona $F$ essi risulta prossimo a 2 .

(*) II numero delle stazioni esaminate era 67 . In hase ad una primissima analisi si scartarono dall'indagine 15 stazioni, 7 perche in zone pianeggianti per larga estensione e 8 perche situate verso la sommita dei principali monti della zona: Monte Amiata, Monte Civitella, Monte Labbro. 
UITERIORI CONSIDERAZIONI SULLA CORREZIONE TOPOGRAFICA, ECC. 297

Bisogna ricordare che il rapporto $A / B=1$ indicherebbe che e verificata la condizione di distribuzione casuale nelle pendenze, per i compartimenti a prevalente variazione radiale delle quote.

Tabella I

\begin{tabular}{|c|c|c|c|c|c|c|c|c|c|}
\hline $\begin{array}{c}\text { Zona } \\
\text { di Hammer }\end{array}$ & $\begin{array}{c}\text { Gr.po } \\
\quad 1\end{array}$ & $\begin{array}{c}\text { Gr.po } \\
\quad 2\end{array}$ & $\begin{array}{c}\text { Gr.po } \\
3\end{array}$ & $\begin{array}{c}\text { Gr.po } \\
4\end{array}$ & $\begin{array}{c}\text { Gr.po } \\
5\end{array}$ & $\begin{array}{c}\text { Gr.po } \\
6\end{array}$ & $\begin{array}{c}\text { Gr.po } \\
\text { A }\end{array}$ & $\begin{array}{c}\text { Gr.po } \\
\text { B }\end{array}$ & $\mathrm{A} / \mathrm{B}$ \\
\hline E & 75 & 32 & 24 & 179 & 22 & 84 & 207 & 50 & 4,14 \\
\hline $\mathbf{F}$ & 31 & 34 & 52 & 143 & 46 & 110 & 186 & 89 & 2,08 \\
\hline G & 75 & 58 & 60 & 177 & 98 & 156 & 236 & 157 & 1,50 \\
\hline $\mathrm{H}$ & 69 & 70 & 122 & 141 & 106 & 116 & 237 & 202 & 1,17 \\
\hline I & 82 & 68 & 76 & 154 & 114 & 130 & 226 & 186 & 1,21 \\
\hline$J$ & 184 & 72 & 78 & 203 & 155 & 140 & 278 & 230 & 1,21 \\
\hline Totale F-I & 441 & 302 & 388 & 818 & 519 & 653 & 1163 & 864 & 1,35 \\
\hline
\end{tabular}

Oltre i risultati dell'analisi globale per le varie zone di tutte le stazioni considerate, si riportano anche i l'isultati dell'analisi per le singole stazioni.

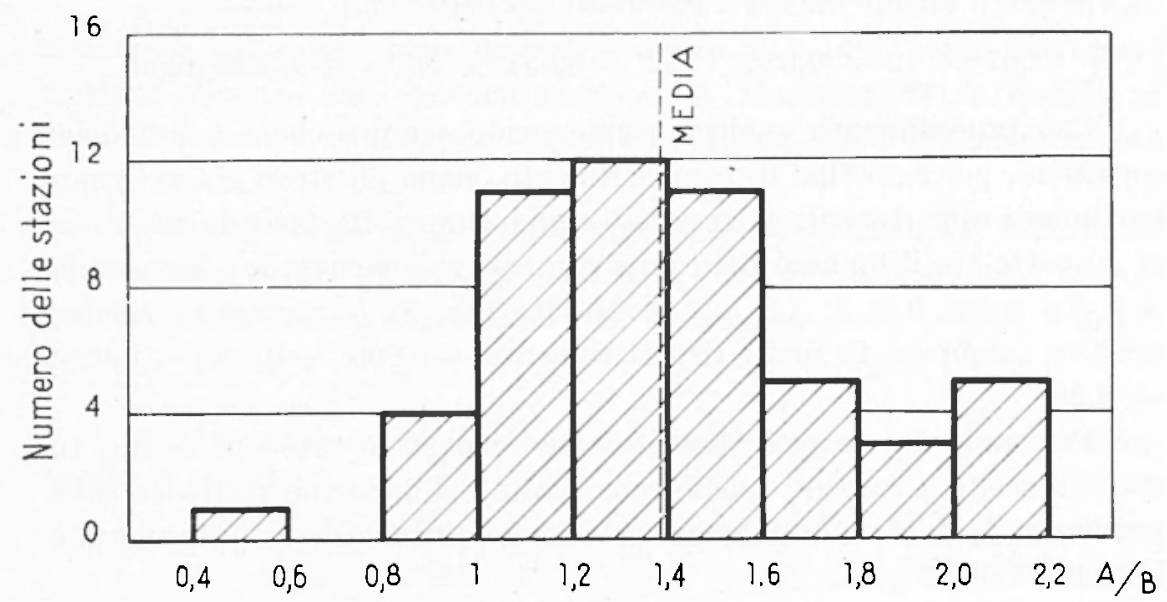

Fig. 5 - Istogramma del numero delle stazioni in funzione del rapporto $A / B$.

Nella Fig. 5 e riportato l'istogramma del numero delle stazioni per le quali il rapporto tra il numero dei compartimenti ascrivibili al gruppo $A$ 
e quello dei compartimenti del gruppo $B$, per il complesso delle zone $F$ - $J$ di Hammer, e compreso nelle classi indicate in ascissa. Si puo notare che per il $75 \%$ delle stazioni il rapporto $A / B$ e compreso tra 1 ed 1,8 e che la media dei rapporti e 1,39 .

Le variazioni del rapporto $A / B$ comportano in media variazioni nell'errore residuo totale nel senso che a rapporti $A / B$ alti (maggiori ad es. di 1,8 ) dovrebbero corrispondere errori resiclui totali negativi, mentre a rapporti $A / B$ bassi (ad es. inferiori di 1,8 ) dovrebbero corrispondere errori residui totali negativi.

Come esempio consideriamo 50 compartimenti di una stazione e supponiamo che il numero dei compartimenti appartenenti a vari gruppi (gruppo $6, A$ e $B$ ) stiano tra loro come il numero totale dei compartimenti appartenenti ai vari gruppi per le zone $F-J$ indicati nella Tabella I.

In tal caso il rapporto $A / B$ e di 1,35 . I compartimenti appartenenti ai vari gruppi sarebbero 12 per il gruppo 6, 22 per il gruppo $A$ e 16 per il gruppo $B$.

Supponiamo che a ciascun compartimento competano errori residui uguali a quelli calcolati per la zona $F$ di Hammer nel caso di pendenze del $25 \%$ (vecli Fig. 3) e $H_{m}$ uguale a $150 \mathrm{~m}$. Per il gruppo 6 l'errore sia pertanto di $+0,007$ mgal, per il gruppo $A-0,036$ mgal e per il gruppo $B+0,05+$ mgal.

L'errore residuo totale sarebbe in tal caso

$$
+0,007 \times 12-0,036 \times 22+0,054 \times 16=+0,150 \mathrm{mgal} .
$$

Con procedimento analogo, supponendo sempre che i valori delle correzioni, per ogni tipo di compartimento, siano gli stessi e che i compartimenti appartenenti al gruppo 6 siano sempre 12 , facendo assumere al rapporto tra il numero dei compartimenti appartenenti ai due gruppi $A$ e $B$ i valori 0,$8 ; 1 ; 1,8 ; 2,2$ (confronta Fig. 5), le correzioni residue relative sarebbero in totale rispettivamente $+0,606 ;+0,426 ;-0,024$; $-0,204$.

Per verificare le osservazioni fatte sono state prese in esame 10 stazioni scelte a caso fra quelle considerate nell'indagine statistica sulle pendenze. L'ubicazione di queste stazioni, contrassegnate da numeri, e indicata nella Fig. 6 .

Per calcolare un valore sufficientemente approssimato della correzione topografica da confrontare con quello ealcolato a mezzo delle tabelle di Hammer, ogni compartimento di Hammer per le zone $F-J$ è stato suddiviso in 16 parti: si è ottenuto cosi una schematizzazione com- 
prendente 960 compartimenti. Sono state valutate le quote medie di ciascuno dei nuovi compartimenti e calcolato il relativo apporto mediante tabelle costruite appositamente (*).

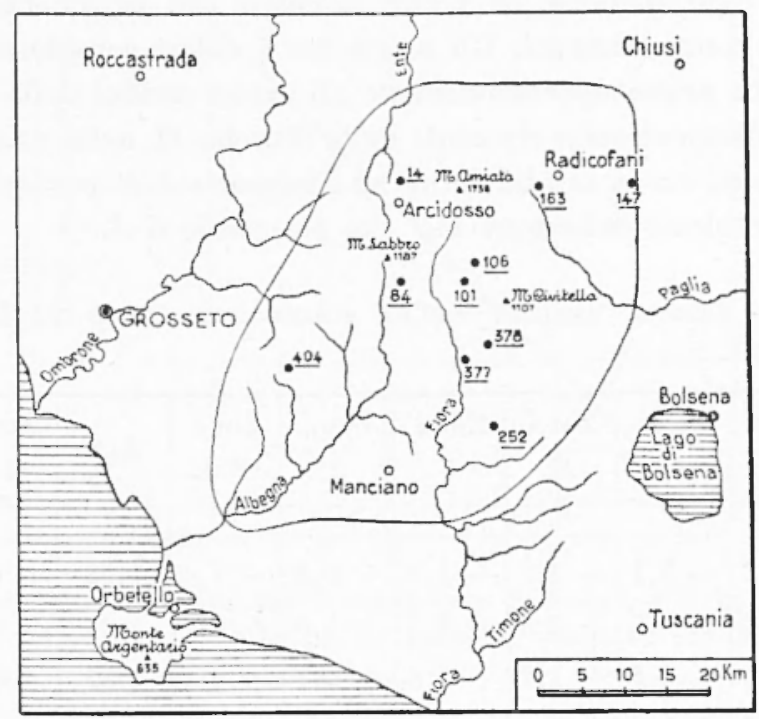

Fig. 6.

La correzione topografica così valutata e ancora affetta da un errore residuo il cui valore pero dorrebbe essere il 5 - $10 \%$ circa dell'errore residuo relativo alla schematizzazione di Hammer (**) e quindi ai fini del presente studio puo essere considerata come sufficientemente approssimata.

La quota media di ciascun compartimento di Hammer e stata ricavata come media aritmetica ponderata delle quote medie dei $16 \mathrm{com}$ partimenti in cui era stato suddiviso, assumendo come pesi le relative

$\left(^{*}\right)$ Si è utilizzata per il calcolo la seguente espressione, ottenuta sviluppando in serie la [3]

$$
1 g=k \sigma\left(a_{2}-a_{1}\right) I_{n}\left[\frac{\Pi_{m}}{R_{1}} \cdot \frac{a-1}{2 a}-\left(\frac{H_{m}}{R_{1}}\right)^{3} \frac{a^{3}-1}{8 a^{3}} \div\left(\frac{\Pi_{m}}{R_{1}}\right)^{5} \frac{a^{5}-1}{16 a^{5}} \cdots\right]
$$

nella quale $\alpha_{2}-\alpha_{1}$ e l'ampiezza angolare del compartimento, $a$ e il rapporto $R_{2} / R_{1}$ tra raggio esterno e raggio interno della zona ed $H_{m}$ la quota media.

(**) L'errore residuo di una schematizzazione si puo considerare in prima approssimazione come inversamente proporzionale al numero dei compartimenti (Vedi appendice I). 
aree. In base a tali quote medie sono state calcolate le correzioni per mezzo delle tabelle di Hammer.

I valori ottenuti sono stati sottratti, sia zona per zona sia globalmente, per ogni stazione, ai valori calcolati con lo schema comprendente i 960 compartimenti. Gli scarti tra i ralori considerati che rappresentano in prima approssimazione gli errori residui della schematizzazione di Hammer sono riportati nella Tabella II nella quale sono indicati anche gli errori residui totali ed i rapporti $A / B$ per ogni stazione, sia per il complesso delle zone $F-J$ che per quello $G-J$.

Tabella II - ERrori RESIdUI dELIA SCIIEMATIZzazione DI HAMMER IN $10^{-2} \mathrm{mgal}$

\begin{tabular}{|c|c|c|c|c|c|c|c|c|c|}
\hline Punto & $\begin{array}{c}\text { Zona } \\
\mathrm{F}\end{array}$ & $\begin{array}{c}\text { Zona } \\
\text { G }\end{array}$ & $\begin{array}{c}\text { Zona } \\
\mathrm{H}\end{array}$ & $\begin{array}{c}\text { Zona } \\
\text { I }\end{array}$ & $\begin{array}{c}\text { Zona } \\
\mathrm{J}\end{array}$ & $\begin{array}{c}\text { Zone } \\
\text { F.J }\end{array}$ & $\mathrm{A} / \mathrm{B}$ & $\begin{array}{c}\text { Zone } \\
\text { G-J }\end{array}$ & $\mathrm{A} / \mathrm{B}$ \\
\hline 14 & $-2,2$ & $-0,1$ & $+1,3$ & $-1,0$ & $+0,6$ & $-1,4$ & 1,92 & $+0,7$ & 1,84 \\
\hline 84 & $+0,4$ & $-0,7$ & $+2,5$ & $+2,7$ & $+1,8$ & $+6,7$ & 1,24 & $+6,8$ & 1,06 \\
\hline 101 & $-2,5$ & $+0,4$ & $+1,7$ & $\div 1,8$ & $+2,7$ & $+4,1$ & 1,32 & $+6,6$ & 1,11 \\
\hline 106 & $+2,1$ & $+2,5$ & $+1,2$ & $+2,1$ & $+0,4$ & $+8,3$ & 1,52 & $+6,2$ & 1,38 \\
\hline 147 & $+1,0$ & $+0,5$ & $+3,2$ & $+2,5$ & $+2,4$ & $+9,6$ & 1,16 & $+8,6$ & 1,12 \\
\hline 163 & $-2,0$ & $+1,4$ & $+1,4$ & $+2,4$ & $+0,3$ & $+3,5$ & 1,83 & $+5,5$ & 1,50 \\
\hline 252 & $+5,2$ & $+1,5$ & $+0,4$ & $+0,4$ & $+0,3$ & $+7,8$ & 1,00 & $+2,6$ & 1,50 \\
\hline 377 & $+2,1$ & $-1,0$ & $\div 0,5$ & $-0,8$ & $+1,1$ & $+3,9$ & 1,69 & $+1,8$ & 2,05 \\
\hline 378 & $+0,3$ & $+0,3$ & $-0,1$ & $+5,1$ & $+1,7$ & $+7,3$ & 1,41 & $+7,0$ & 1,30 \\
\hline 404 & $-7,6$ & $+1,4$ & 0 & $+2,1$ & $+0,3$ & $-3,8$ & 2,20 & $+3,8$ & 1,70 \\
\hline Medie & $-0,3$ & $+0,6$ & $+1,2$ & $+1,7$ & $+1,2$ & & & & \\
\hline
\end{tabular}

I risultati riportati nella Tabella confermano le considerazioni fatte nel paragrafo 3 ; in particolare si puo notare:

1) la massima parte degli errori residui, presi sia singolarmente per ogni zona e per ogni stazione, sia globalmente, sono positivi;

2) se si considerano le varie zone, i valori negativi sono piu frequenti nelle zone piu vicine alle stazioni ( 4 valori per la $F$ e 3 per la $G$ ) mentre sono assenti nella zona $J$;

3) confrontando la media per le varie zone riportate in fondo alla Tabella II con le medie corrispondenti dei rapporti $A / B$ della Tabella I 
ULTERIORI CONSIDERAZIONI SULLA CORREZIONE TOPOGRAFICA, ECC. 301 $(2,08$ per la $F, 1,50$ per la $G$ e 1,$17 ; 1,21 ; 121$ rispettivamente per la $H$, $I, J)$ si puo osservare che si verifica una correlazione inversa tra rapporti $A / B$ ed errori;

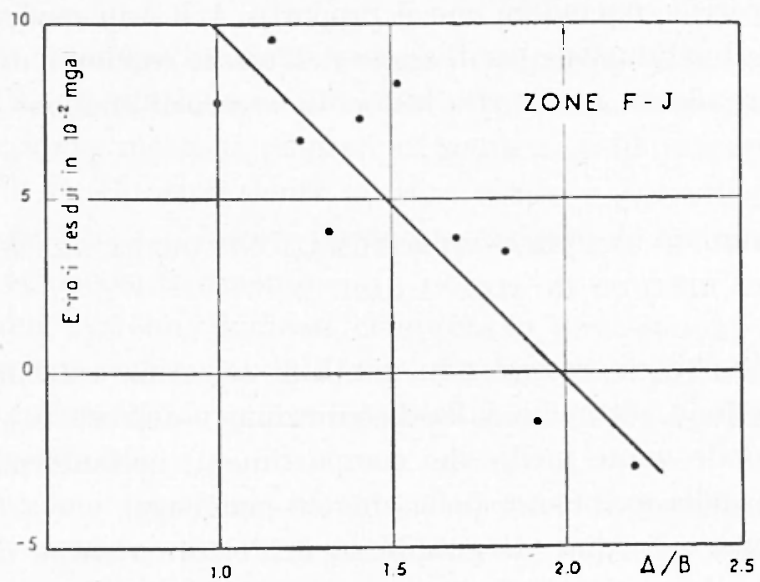

Fig. 7 - Errori residui per li complesso dellezone $F-J$ in funzione del rapporto $A / B$ per le 10 stazioni prese in considerazione e relativa retta di regressione.

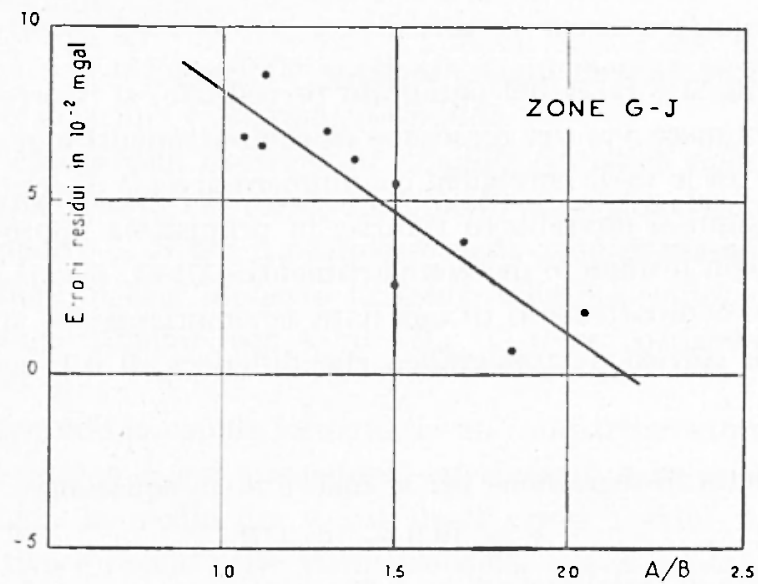

Fig. 8 - Errori residui per il complesso delle zone $G-J$ inf unzione del rapporto $A / B$ per le 10 stazioni prese in considerazione e relativa retta di regressione.

4) anche considerando i valori relativi alle singole stazioni per i complessi delle zone $F-J$ e $G-J$, si riscontra la stessa correlazione inversa. 
Nelle Figg. $i$ e 8 sono riportati in diagramma gli errori residui di ogni stazione in funzione del rapporto $A / B$ separatamente per le zone $F-J$ e $G-J(*)$.

Si puo percio conchudere che il rapporto $A / B$ a in media un indice significativo di valutazione per il segno dell'errore residuo e di confronto, a parita di pendenze medie, fra valori di errori di stazioni diverse.

\section{5. - Valutazioxf di Prima APprossimazione DELLA GRANDEZZA DEL- L'FRRORE RESIDUO IN ALCUNI CASI.}

Si e visto che gli errori residui totali di una zona o di un complesso di zone dipendono, oltre che dalla distribuzione e dell'orientamento delle pendenze e delle quote medie dei compartimenti nell'intorno della stazione, anche dalla grandezza delle singole pendenze.

Per valutare l'ordine di grandezza dell'errore residuo occorre confrontare, per aleune stazioni prese come campioni, i valori delle correzioni ealcolate per mezzo della scelematizzazione in esame con $\mathrm{m}$ valore sufficientemente approssimato della correzione stessa.

Quest'ultimo si puo calcolare seguendo ad es. due metodi semirigorosi:

1) come si e fatto nel palagrafo precedente, si ricorre a suddivisioni in un numero via via crescente di compartimenti e si controllano le variazioni tra le varie correzioni con numero diverso di compartimenti. Gli errori residui si dorrebbero pidure, in primissima approssimazione, linearmente con il numero dei compartimenti. Ad es. se quadruplieando il numero dei compartimenti di una data schematizzazione si ottiene un valore per la correzione topografica che differisce di 0,1 mgal rispetto

(*) La retta di regressione per le zone $F-J$ ha equazione

$$
\varepsilon=19,9-10 A / B
$$

ed il coefficiente di correlazione e $-0,87$

Per le zone $G-J$ si ha analogamente

$$
\varepsilon=15,0-6,9 A / B
$$

ed il coefficiente di correlazione e $-0,88$

I.e correlazioni sono significative. Gli scarti rispetto alla retta di regressione sono doruti, e a condizioni topografiche particolari, e alla influenza dei compartimenti a variazione angolare delle quote, che non compaiono nei rapporti $A / B$ ed i cui errori residui, come gia visto, sono positivi. 
al valore ottenuto in base alla schematizzazione iniziale, l'errore della schematizzazione iniziale sara in prima approssimazione uguale a

$$
0,1 \cdot 4 / 3=0,13 \mathrm{mgal} \text {. }
$$

2) Si prendono in esame per ogni compartimento i tre parametri: quota media, orientazione e valore della pendenza media, e si calcolano gli errori residui a mezzo di diagrammi simili a quelli indicati nella Fig. 3 o a mezzo di abachi equivalenti, preventivamente costruiti per ogni zona e per ogni pendenza. Iserrore residuo totale sara la somma degli errori residui di ogni compartimento.

Ambedue i metodi indicati comportano procedimenti lunghi e laboriosi; per una valutazione di larga massima, che nella maggior parte dei casi è sufficiente, si puo ricorrere a metodi piu speditivi anche se meno approssimati.

In situazioni specifiche, in cui la superficie topografica in un certo intorno alla stazione sia rapplesentabile con superfici semplici, si può ricorrere ad espressioni particolari quali ad es. quelle gia viste per stazioni situate sulla sommita di un rilievo o quelle ricavate da C. IT. Sandberg $\left({ }^{8}\right)$ per stazioni situate sul fondo o sui fianchi di vallate a $\mathrm{V}$.

Non appena si considerano zone piu lontane nelle quali le pendenze e le quote medie variano da compartimento a compartimento in modo irregolare, non e piu possibile applicare alcun metodo semirigoroso che sia nello stesso tempo relativamente rapido.

In tal caso si puo ricorrere all'indagine statistica sulla orientazione delle pendenze svolta nel paragrafo precedente e prendere in considerazione il rapporto $A / B$ per il complesso delle zone in esame.

Un valore di tale rapporto prossino all'unita indica che in media ad ogni compartimento con $\operatorname{tg} \beta \cdot H_{m}>0$ ne corrisponda uno con $\operatorname{tg} \beta \cdot H_{m}<0$.

Considerando le coppie formate da un compartimento del gruppo $A$ ed uno del gruppo $B$ cui competono valori simili delle quote $H_{m}$ e delle pendenze $\operatorname{tg} \beta$, la media dei ralori degli errori residui '’, come si e detto, positiva e ricade nelle vicinanze della curva relativa ai compartimenti con variazione angolare delle quote con $\operatorname{tg} \delta=|\operatorname{tg} \beta|$.

Anzi, se si considerano le curve delle medie tra gli errori dei compartimenti dei gruppi $A$ e $B$ al variare dei rapporti $A / B$ (Fig. 9), si puo notare che, con sufficiente approssimazione, si puo sostituire la media degli errori con i valori del corrispondente errore per compartimenti a variazione angolare delle quote nei casi di rapporti $A / B$ compresi tra 1 e 1,4 . Per $A / B=1,2$, in media $\mathrm{i}$ due valori sono molto prossimi. 
In tal caso si potrebbe calcolare l'errore residuo totale considerando per ciascun compartimento, anche se a prevalente variazione radiale della quota, l'errore che si avrebbe in un compartimento a variazione angolare della quota con stessa quota media e stessa pendenza.

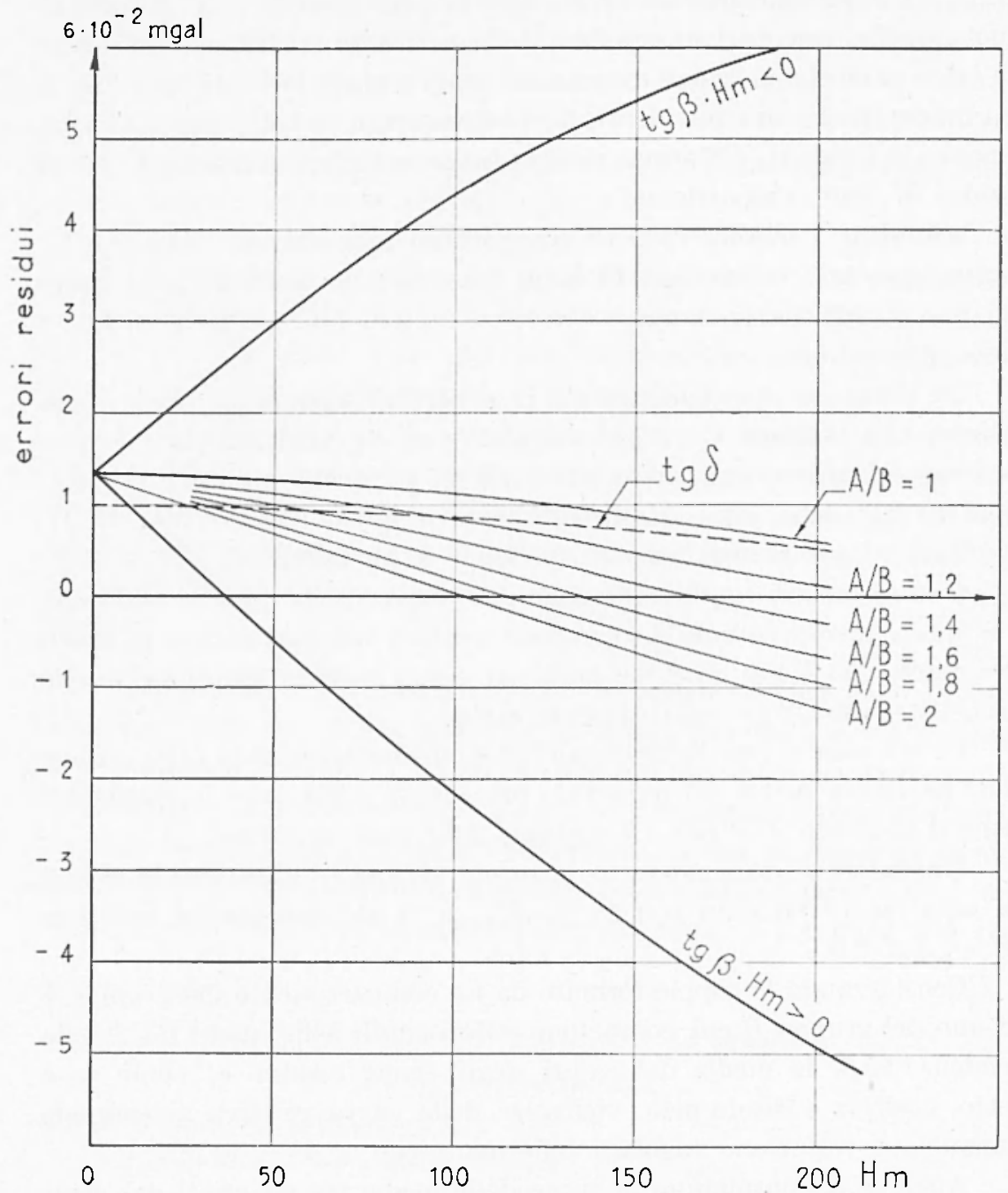

Fig. 9 - Frrori residui medi dei compartimenti della zona $F$ di Ilammer a variare del rapporto $A / B$ per pendenze di 0,25 .

Nel calcolo risulterebbe cosi eliminato uno dei parametri che entrano nella valutazione semirigorosa dell'errore: la orientazione della pendenza della superficie topografica. 
Se i l'xpporti $A / B$ sono superiori ad $1,3-1,4$, i valori dell'errore ottemuti applicando il metodo sopra proposto, semebbero magrenioni di quelli ellettivi; essi fornirebbero quindi un limite massimo dell'entita dell'errore elfettixo stesso.

Il metodo indicato dunque puo portare, secondo i casi, ald un valore notevolmente approssimato dell'errore (e permettere quindi la sua eliminazione, se necessaria) o quanto meno a dare utili indicazioni di larga massima.

Esso pero comporterebbe la costruzione prima e l'utilizzazione poi di diagrammi o abachi a doppia entrata (to $\delta$ ext $H_{m}$ ) di scomoda lettura.

Per semplificare i calcoli si è introdotta quindi la funzione

$$
\eta=\frac{1}{6}\left(g_{H_{\max }}+4 g_{H_{m}}+g_{H_{\min }}\right)
$$

in cui $y_{\|}$e la funzione [3] ricondata calcolata per le quote relative massima, media e minima del compartimento. Si dimostra (vedi appendi(e $\mathrm{II}^{\circ}$ ) rhe tale funzione si approssima alla funzione [:2], che fornisce lattrazione di un compartimento con variazioni angolari delle quote, con scarti che solo ecerezionalmente superano a $10^{-6}$ gal.

L'errore residuo dovuto alla schematizzazione in compartimenti cilindriei piani nel caso di variazione angolare delle quote, puo essere pertanto calcolata mediante l'espressione: (*)

$$
\begin{gathered}
\varepsilon_{\eta}=\frac{1}{6}\left(g_{H \max }+4 g_{H m}+g_{H_{\min }}\right)-g_{H_{m}}= \\
=\frac{1}{6}\left(g_{H_{\max }}-2 g_{H_{m}}+g_{H_{\min }}\right)
\end{gathered}
$$

Il calcolo della [4] e della [5] e semplice e non richiede l'uso di abachi o di diagrammi particolari, in quanto utilizza le normali tabelle di correzione.

Orviamente l'espressione [4] ha le stesse limitazioni della fumzione [थ] per la valutazione dell'erore globale di un gruppo di zone nel caso piu gremerale.

Il valore della correzione calcolata con tale formula sara pereio a sua volta affetta da un errore residuo che dipendera dal valore di $A B$, a parita di altre condizioni.

(*) L'espressione [5] e equivalente alla formula ricavata da .H. C'mieti ( ${ }^{3}$ ) per il caleolo degli errori resitui per zone con variazione angolare thelle quote. 
Per portare un esempio numerico si sono calcolati gli errori residui dovati all'uso della [4] per le 10 stazioni considerate nel precedente paragrafo, relativamente ai complessi delle zone $F-J$ e $G-J$ della configurazione di Hammer.

Come valori di confronto, si sono considerati quelli ricavati con la suddivisione in 960 compartimenti Nella Tabella III sono indicati i risultati dei calcoli: si riportano ordinatamente per ogni complesso di zone il rapporto $A / B$, l'errore residuo della suddivisione di Hammer (gia indicato nella Tabella II) e l'errore residuo dovuto all'esporessione [t].

Tabella III

\begin{tabular}{|r|r|r|r|r|r|r|}
\hline \multirow{2}{*}{ Stax. } & \multicolumn{3}{|c|}{ Z one F.J } & \multicolumn{3}{|c|}{ Z one G.J } \\
\cline { 2 - 6 } & A/B & er. IIam. & er. F(4) & A/B & er. IIam. & er. F(4) \\
\hline & & & & & & \\
\hline 14 & 1,92 & $-1,4$ & $-9,3$ & 1,84 & $+0,7$ & $-5,8$ \\
84 & 1,24 & $+6,7$ & $-2,2$ & 1,06 & $+6,8$ & $-1,3$ \\
101 & 1,32 & $+4,1$ & $-4,8$ & 1,11 & $+6,6$ & 0 \\
106 & 1,52 & $+8,3$ & $-2,2$ & 1,38 & $+6,2$ & $-0,3$ \\
147 & 1,16 & $+9,6$ & $-0,5$ & 1,12 & $+8,6$ & $+1,6$ \\
163 & 1,83 & $+3,5$ & $-3,6$ & 1,50 & $+5,5$ & $-0,2$ \\
252 & 1,00 & $+7,8$ & $+1,7$ & 1,50 & $+2,6$ & $+0,7$ \\
377 & 1,69 & $+3,9$ & $-3,8$ & 2,05 & $+1,8$ & $-4,6$ \\
378 & 1,41 & $+7,3$ & $-2,9$ & 1,30 & $+7,0$ & $+0,1$ \\
404 & 2,20 & $-3,8$ & $-7,3$ & 1,70 & $+3,8$ & $+1,0$ \\
& & & & & & \\
\hline
\end{tabular}

Nella Tabella III si puo notare che per rapporti $A / B$ superiori a 1,8 l'errore residuo dovuto all'applicazione della [4] e maggiore e talvolta anche di molte volte, a quello dovuto alla sehematizzazione di Hammer.

In questo caso dunque l'errore residuo calcolato con la formula [5] fornirebbe valori molto superiori a quelli che si avrebbero applicando i metodi semirigorosi. Si avrebbe cioe solo una valutazione del limite massimo dell'entita dell'errore.

Nel caso di rapporto $A / B$ compreso tra 1,8 e 1,6 (punto 377 zone $F-J)$ gli errori residui- sono, nei due casi, quasi uguali ma di segno op- 
posto. I'errore residuo calcolato con la [5] avrebbe pertanto nul valore doppio di quello effettivo.

Per valori $A / B$ inferiori ad 1,6, se si accettua il numero 101 per le zone $F-J$, i valori degli errori residui dovuti all'espressione [4] sono quasi sempre inferiori di oltre il $30 \%$ a quelli dovnti alle schematizzazioni di Hammer.

In questi casi l'applicazione della [5] porta risultati sufficientemente approssimati, tanto da permettere mat correzione dellerrore stesso, se necessario.

Per l'uso della [5] e necessario leggere sulle carte topografiche, oltre la quota media, anche le quote massime e minime, limitatamente pero a quei compartimenti il cui dislivello massimo superi valori tali da fornire correzioni per l'errore residuo superiori a grandezze prefissate.

Tali valori possono essere ad es. ricavati dai diagrammi di Fig. 4, o analoghi, ma rolta stabilita l'entita massima per l'errore residuo tollerabile per un compartimento. E ovvio che nel fissare tale entita bisogna temer conto del fatto che l'errore residno è in media sistematico.

Nei casi in ani i rapporti $A / B$ siano sufficientemente bassi, il metodo proposto risulta notevolmente rapido e permette di ottenere le stesse approssimazioni di sistemi molto piil complianti.

Desidero ringraziare il Prof. Carmelo Aquilina, Direttore dell'Istituto di Geofisica Mineraria che e stato prodigo di suggerimenti e consigli ed i colleghi Antonio Bossola e Claudio Zacchi che hanno collaborato nei alcoli.

\section{$\triangle P$ PE N J I C E I}

ERrorI RESIDUI IN FUNZIONE DEL NUMERo DET COMPARTMENTT.

Supponiamo di suddividere un compartimento cilindrico in $n^{2}$ parti mediante la suddivisione sia in $n$ parti dell'ampiezza angolare del compartimento $\gamma$, sia dell'intervallo $R_{2}-R_{1}$.

Prendiamo in esame il caso di quote variabili linearmente con la anomalia; la funzione [2] del testo sviluppata in serie da:

$$
\begin{gathered}
y=K \sigma \gamma^{\prime}|R| \frac{1}{2}\left(\frac{H_{m}}{R}\right)^{2}+\frac{1}{6}\left(\frac{D}{2}\right)^{2}-\frac{1}{8}\left(\frac{H_{m}}{R}\right)^{4}-\frac{1}{4}\left(\frac{H_{m}}{R}\right)^{2}\left(\frac{D}{2 R}\right)^{2}- \\
-\frac{1}{40}\left(\frac{D}{2 R}\right)^{4}+\frac{1}{16}\left(\frac{H_{m}}{R}\right)^{6}+\frac{\partial}{16}\left(\frac{H_{m}}{R}\right)^{4}\left(\frac{D}{2 R}\right)^{2}+\frac{3}{16}\left(\frac{H}{R}\right)^{2}\left(\frac{D}{2}\right)^{4}+ \\
\left.\left.+\frac{1}{7 \cdot 16}\left(\frac{D}{2 R}\right)^{6}+\ldots\right\}\right]_{R_{2}}^{R_{1}}
\end{gathered}
$$


l'espressione [3] del testo della attazione di un compartimento di quota $H_{m}$ sviluppata in serie a:

$$
g_{H_{m}}=K \sigma \gamma^{\prime}\left|R\left(\frac{1}{2}\left(\frac{H_{m}}{R}\right)^{2}-\frac{1}{8}\left(\frac{H_{m}}{R}\right)^{4}+\frac{1}{16}\left(\frac{H_{m}}{R}\right)^{6}-\ldots\right\}\right|_{R^{2}}^{R^{1}}
$$

La differenza tra le due esplessioni[ 1] e [2] e:

$$
\begin{aligned}
A g=K \sigma \gamma[R & \frac{1}{6}\left(\frac{D}{2 R}\right)^{2}-\frac{1}{4}\left(\frac{H_{m}}{R}\right)^{4}\left(\frac{D}{2 R}\right)^{2}-\frac{1}{40}\left(\frac{D}{2 R}\right)^{4}+\frac{5}{16}\left(\frac{H_{m}}{R}\right)^{2}\left(\frac{D}{2 R}\right)^{2} \\
& \left.+\frac{3}{16}\left(\frac{H_{n}}{R}\right)^{2}\left(\frac{D}{2 R}\right)^{4}+\frac{1}{7 \cdot 16}\left(\frac{D}{2 R}\right)^{6}+\ldots\right\}\left.\right|_{R_{2}} ^{R_{1}}\left({ }^{*}\right)
\end{aligned}
$$

Per una valutazione dell'ordine di grandezza dell'erorore consideriamo solo il primo termine della serie [3].

Ricordando che $D=\gamma R_{m} \operatorname{tg} \delta$

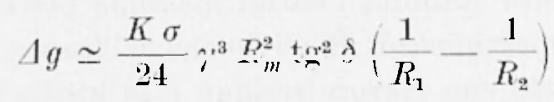

con l'applossimazione $R_{1} \cdot R_{2} \simeq R_{m}^{2}$ l'esplessione sopura seritta diventa:

$$
\Delta \eta=\frac{K \sigma}{2 t} \gamma^{3} \operatorname{tg}^{2} \delta\left(R_{2}-R_{1}\right)
$$

L'errore di uno degli $n$ - nuovi compartimenti di ampiezza angolare $\gamma / n$ limitati tra due circonferenze distanti $\left(R_{2}-R_{1}\right) / n$ sara dato da:

$$
\Delta g^{\prime} \simeq \frac{K a}{2 t} \frac{\gamma^{3} \operatorname{tg} g^{2} \delta\left(R_{2}-R_{1}\right)}{n^{4}}
$$

Poiche nel caso considerato gli errori sono tutti sistematicamente positivi, l'errore del complesso degli $n$ - nuovi compartimenti sara dato da:

$$
\Sigma \Delta g^{\prime} \simeq \frac{K \sigma}{24} \quad \frac{\gamma^{3} t_{g^{2}} \delta\left(R_{2}-R_{1}\right)}{n^{2}}
$$

Confrontando la [5] con la [4], si puo concludere che in prima approssimazione la suddivisione di un compartimento in $n$ - compartimenti minori porta ad una riduzione dell'errore di $n$ - volte, cio signifiea che l'errore descresce linearmente con l'ammento del numero dei compartimenti.

(*) La serie [3], limitata ai primi tre termini coincide con la espressione della correzione per distribuzione angolare ricavata da M. Cunieti ${ }^{3}$ ). 


\section{A P P E N I C E II}

La funzione

$$
\eta=\frac{1}{6}\left(g_{H_{\max }}+4 g_{H_{m}}+g_{H_{\mathrm{m} \mathbf{n}}}\right)
$$

in cui $g_{H}$ è la funzione [3] del testo calcolata per le quote relative massima media e minima del comparitmento, sviluppata in serie da:

$$
\begin{gathered}
\eta=K \sigma \gamma\left[R \left(\frac{1}{2}\left(\frac{H_{m}}{R}\right)^{2}+\frac{1}{6}\left(\frac{D}{2 R}\right)^{2}-\frac{1}{8}\left(\frac{H_{m}}{R}\right)-\frac{1}{4}\left(\frac{H_{m}}{R}\right)^{2}\left(\frac{D}{2 R}\right)^{2}-\right.\right. \\
-\frac{1}{24}\left(\frac{D}{2 R}\right)^{4}+\frac{1}{16}\left(\frac{H_{m}}{\mathrm{R}}\right)^{6}+-\frac{5}{16}\left(\frac{H_{m}}{R}\right)^{4}\left(\frac{D}{2 R}\right)^{2}+\frac{\mathrm{b}}{16}\left(\frac{H_{m}}{R}\right)^{2}\left(\frac{D}{2 R}\right)^{4}+ \\
\left.\left.-+\frac{1}{16}\left(\frac{D}{2 R}\right)^{6}+\ldots\right\}\right]_{R_{2}}^{R_{1}}
\end{gathered}
$$

Sottraendo l'espressione su scritta alla [1] dell'appendice I che fornisce il valore esatto della correzione topografica nel caso di variazione lineare della quota con la anomalia, si ha:

$$
\left.\Delta=K \sigma \gamma\left[\frac{1}{8} R\left(\frac{D}{2 R}\right)^{4}, \quad \frac{2}{15}+\left(\frac{H}{R}\right)^{2}-\frac{3}{7}\left(\frac{D}{2 R}\right)^{2}+\ldots\right\}\right]_{R_{2}}^{R_{1}}
$$

Tale differenza e funzione dei termini di grado elevato delle funzioni sopra viste, che, nei casi interessanti il presente studio, convergono rapidamente. Per avere un ordine di grandezza massimo del valore di tale differenza, si può considerare la zona $F$ di Hammer per la quale e massimo il rapporto $R_{2} / R_{1}$ : per tg $\delta=0,50$ ed $H_{m}=200 \mathrm{~m}$ la differenza $\mathrm{e}$ di $1,02 \cdot 10^{-6}$ gal.

\section{BIBLIOGRAFIA}

(1) M. BERNABINI, Sulla correzione topografica nei rilievi gravimetrici geo. minerari. "Annali di Geofisica" XV, 1, (1963).

(2) G. Cassinis, P. Dore, S. Ballarin, Tavole fondamentali per la riduzione dei valori osservati della gravita. Pubblicazione della $\mathrm{R}$. Commissione Geodetica Italiana, Nuova serie n. 13 (1937).

(3) M. Cunietti, Natura ed entita degli errori nelle riduzioni topografiche ed isostatiche dei valori della gravita. "Annali di Geofisica"1-4, (1948). 
(4) F. Falini, Notizie preliminari su una campagna di indagini e ricerche per minerali di mercurio nella regione del $M$. Amiata. Periodico di Mineralogia XXXIX, 1, (1960).

(5) S. HAMmeR, Terrain correction for gravimetric stations. "Geophysics" IV, 3, (1939).

${ }^{\left({ }^{6}\right)}$ M. K. KUBERT, Gravitational terrain effects for two-dimensional topographic features. "Geophysics" XIII, 2, (1948).

(') P. LEJax, Developpements modernes de la gravimetrie. Gauthier-Villars, Paris 1947.

$\left.{ }^{8}\right)$ C. H. SANDBERG, Terrain correction for an inclined plane in gravity computations. "Geophysies" XXIII, 4, (1958).

( $)$ L. SoraINI, Sulla riduzione topografica delle misure di gravita. "Rivista Geomineraria " 2-3, 1943. 